\title{
DETECTION AND IDENTIFICATION OF DARK MATTER
}

\author{
DAVID G. CERDEÑO \\ Departamento de Física Teórica C-XI, and Instituto de Física Teórica UAM-CSIC, \\ Universidad Autónoma de Madrid, Cantoblanco, E-28049 Madrid, Spain \\ cerdeno@delta.ft.uam.es
}

\begin{abstract}
Dark matter is an abundant component of our Universe and its detection and identification constitutes one of the most challenging goals in modern Physics. Particle Physics provides well motivated candidates for dark matter, among which a generic weaklyinteracting massive particle (WIMP) stands out for its simplicity and the fact that WIMP candidates can be found in many theories proposing new physics at the $\mathrm{TeV}$ scale, such as Supersymmetry, models with Universal Extra Dimensions and Little Higgs Theories. I will review the properties of some of the main WIMP candidates and their detectability (with special emphasis on direct detection experiments). I will also address the strategies that can be used to discriminate among them in the case of a future detection.
\end{abstract}

Keywords: Dark matter; theories beyond the standard model, extra dimensions, supersymmetry.

PACS Nos.: 95.35.+d, 12.60.-i, 11.10.Kk, 12.60.Jv

\section{Introduction}

Astrophysical observations performed at various scales of the Universe reveal gravitational effects which cannot be accounted for by the mass contained in the luminous objects. For example, measurements of peculiar velocities or weak lensing effects in clusters of galaxies can only be understood if these systems contain much more matter than the one that emits light. Similarly, the flatness of the rotation curves of spiral galaxies cannot be explained by the luminous galactic disk and leads to hypothesize the existence of an extensive halo of dark matter around them. Recently, the studies of the Bullet cluster have provided further evidence supporting the need for this exotic kind of matter.

Moreover, the observations of the Cosmic Microwave Background (CMB) have allowed the determination of the cosmological parameters with unprecedented precision. Combining the results obtained by the WMAP satellite with those coming from observations of baryon acoustic oscillations and supernovae the relic abundance of dark matter (expressed in units of the critical density) is found to be $\Omega h^{2}=0.1143 \pm 0.0034 .^{1}$

Dark matter is thus an abundant component of our Universe, and its detection and identification is currently of the most challenging goals in modern Physics. This 
problem, once considered a purely astrophysical question, is now deeply related to Particle Physics, since it is within the context of theories describing Physics beyond the Standard Model (SM) that the most plausible candidates for dark matter arise.

Viable dark matter candidates have to fulfil a number of requisites. They must be non-baryonic, non-relativistic, stable on cosmological scales, and neutral (since otherwise they would have been already observed in searches for exotic isotopes). Also, they must reproduce the observed value of the relic abundance, while not being in conflict with Big Bang Nucleosynthesis or stellar evolution.

Particle Physics provides good dark matter candidates, appearing in well motivated extensions of the SM. In this sense, a generic stable particle with Electroweakscale interactions (WIMP) is a very natural solution to the problem of dark matter, because it would be thermally produced in the early Universe in a sufficient number to account for a significant fraction of the relic matter density. Since no new interaction scale is needed, WIMPs can easily be obtained in theories for new Physics at the $\mathrm{TeV}$ scale. Such is the case of Supersymmetric scenarios, Universal Extra Dimension models and Little Higgs Theories. Likewise, a bottom-up approach can be adopted in which minimal additions to the SM are considered, involving the inclusion of a WIMP field (usually a singlet) and new symmetries that protect their decay (in some cases, also a mediator sector that couples the WIMP to the SM).

It should finally be said that WIMPs are not the only solution to the problem of dark matter. Other interesting possibilities include axions, superweakly-interacting massive particles (such as the gravitino and axino in supersymmetric models or the Kaluza-Klein graviton in models with extra dimensions), and decaying dark matter (with a lifetime larger than the age of the Universe).

\section{WIMP Detection}

There are various strategies to attempt the detection of dark matter particles, namely direct, indirect and collider searches. Interestingly, the three of them can be sensitive to WIMP candidates.

Direct detection. If WIMPs constitute the bulk of the dark matter, they would cluster gravitationally with ordinary stars, forming galactic halos. This implies that there is a flux of these particles crossing the Earth, raising the hope of detecting them directly through their elastic interaction with nuclei inside a detector. The resulting nuclear recoils can be observed through ionization, scintillation or an increase of the temperature, and modern experiments use a combination of these signals in order to reduce the background more effectively. At the microphysics level, the interaction of a WIMP with quarks can be described in terms of an effective Lagrangian, which can contain in general scalar, vector and axial couplings (depending on the nature of the WIMP). When the total WIMP-nucleus cross section is calculated, the scalar and vector couplings contribute to the spin-independent (SI) part of the cross section, which is proportional to the square of the total number of nucleons, $A^{2}$. On the other hand, the axial coupling gives rise to a spin-dependent (SD) contribution 
which is a function of the WIMP spin and the nucleus angular momentum. The spin-independent cross section is the dominant contribution for nuclei with $A>20$, as is generally the case in the targets used in dark matter experiments.

The experimental situation regarding direct detection of WIMPs is very exciting. The DAMA/LIBRA collaboration, who investigates a possible annual modulation in the nuclear recoils on sodium iodine (NaI) as a signal for dark matter, claims to have data compatible with the observation of dark matter. ${ }^{2}$ If interpreted in terms of WIMPs, it would correspond to particles with a mass in the $10-100 \mathrm{GeV}$ range and a scattering cross section of order $\sigma^{S I} \approx 10^{-4}-10^{-6} \mathrm{pb}$. This signal has, however, not been confirmed by other detectors and collaborations such as $\mathrm{CDMS}^{3}$ (using Ge and Si crystals), XENON10 ${ }^{4}$ (based on liquid Xe), CoGeNT ${ }^{5}$ (running a low-threshold Ge experiment), and COUPP $^{6}$ and $\mathrm{KIMS}^{7}$ (with targets sensitive to also the spin-dependent contribution) provide stringent experimental upper bounds on the WIMP-nucleus scattering cross section. It should be noted that attempts exist to explain the DAMA/LIBRA signal in terms of less conventional dark matter (e.g., inelastic scattering or very light axion-like particles). In any case, future experiments (such as SuperCDMS or XENON1T) will be able to explore values of the spin-independent scattering cross section up to $\sigma^{S I} \sim 10^{-10} \mathrm{pb}$, thus probing many models for WIMP dark matter.

Indirect detection. Dark matter can also be searched for indirectly, attempting to detect the products of its annihilation in the dark matter halo. These include gamma rays (which can be observed in imaging atmospheric Cerenkov telescopes like MAGIC and space-based gamma-ray detectors such as EGRET and Fermi), neutrinos (from WIMP annihilation inside the Sun or Earth and which can be detected through the resulting up-going muons in neutrino telescopes such as Amanda, SuperKamiokande, BAIKAL and the future IceCube, ANTARES and KM3Net) and antimatter (such as positrons, antiprotons or antideuterons, which can be observed in space-based experiments such as AMS and PAMELA). In fact, the recent results reported by the PAMELA collaboration ${ }^{8}$ indicate an excess in the positron flux but no excess in the antiproton flux. This, if interpreted in terms of dark matter, disfavours some WIMP models such as the neutralino (whose annihilation into a pair of fermions is helicity suppressed, being a Majorana fermion) and may favour some others (such as Kaluza-Klein dark matter). However a very large boost factor is generically needed. Finally, there might also be an astrophysical interpretation of the signal (see, e.g., Ref. 9).

Collider searches. Finally, WIMPs may be copiously produced at colliders such as the future LHC or ILC. Although these cannot provide a final evidence that the detected WIMP is the dark matter of the Universe, they can precisely determine some of the WIMP properties, especially its mass (and to a smaller extent, also its cross section). Thus, the information from these experiments can be combined with the results from direct and indirect searches.

The extraordinary advances in the detection techniques (both direct and indirect), together with the forthcoming start of the Large Hadron Collider, will not 
only allow us to probe different models for dark matter, but also will provide complementary information with which we may be able to discriminate among the various candidates.

\section{Theoretical Predictions for WIMP Direct Detection}

Let us briefly review the theoretical predictions for the direct detection of WIMPs. We will concentrate on those candidates which arise in well-motivated theories.

\subsection{Supersymmetric theories}

In Supersymmetric (SUSY) extensions of the SM a discrete symmetry, known as $R$-parity, is often imposed in order to forbid lepton and baryon violating processes which could lead, for instance, to rapid proton decay. An interesting phenomenological implication is that SUSY particles can only be produced or destroyed in pairs, thus rendering the lightest SUSY particle (LSP) stable.

In large areas of the parameter space of SUSY models, the LSP is an electrically neutral particle, the lightest neutralino, $\tilde{\chi}_{1}^{0}$, which therefore constitutes a very well motivated DM candidate, within the class of WIMPs. ${ }^{10,11}$ The neutralino is a linear superposition of the fermionic partners of the neutral electroweak gauge bosons (bino and wino) and of the neutral Higgs bosons (Higgsinos), and the resulting detection cross section is extremely dependent on its specific composition. More specifically, within the framework of the Minimal Supserymmetric Standard Model (MSSM) the SI part of the neutralino-nucleon cross section receives contributions from Higgs exchange in a $t$-channel and squark exchange in an $s$-channel. ${ }^{15,14,12,13}$ The latter also contributes to the SD part of the cross section, together with a $Z$ boson exchange in a $t$-channel. Thus, a large Higgsino component induces an enhancement of both the Higgs and $Z$ boson exchange diagrams, thereby leading to an increase in both the SD and SI cross sections. On the other hand, the presence of light squarks (if they are almost degenerate with the neutralino) can lead to an enhancement of (mainly) the SD cross section.

Analyses of general supersymmetric scenarios with parameters defined at low energy reveal that the neutralino SI cross section can be as large as $10^{-5} \mathrm{pb}$ for a wide range of neutralino masses up to $\mathcal{O}(1 \mathrm{TeV}) .{ }^{17,18}$ When gaugino masses do not fulfil the GUT relation, very light neutralinos with masses $m_{\chi_{1}^{0}} \gtrsim 7 \mathrm{GeV}$ and large cross-section can be obtained. ${ }^{19,20}$ These results coincide with those obtained in Supergravity theories, where the SUSY parameters are defined at the GUT scale. In the simplest case where the soft parameters defining the model have a universal structure both the SI and SD cross-section are generally small, $\sigma^{S I} \approx \mathcal{O}\left(10^{-8} \mathrm{pb}\right)$, and $\sigma^{S D} \approx \mathcal{O}\left(10^{-4} \mathrm{pb}\right)$. In more general Supergravity scenarios the predicted crosssection can be significantly larger through the inclusion of non-universal values for either the scalar masses (non-universalities in the Higgs mass parameters being the most effective), the gaugino masses or both (see, e.g., Ref. 21 and references therein). The resulting predictions for both the SI and SD contributions are shown on Fig. 1. 

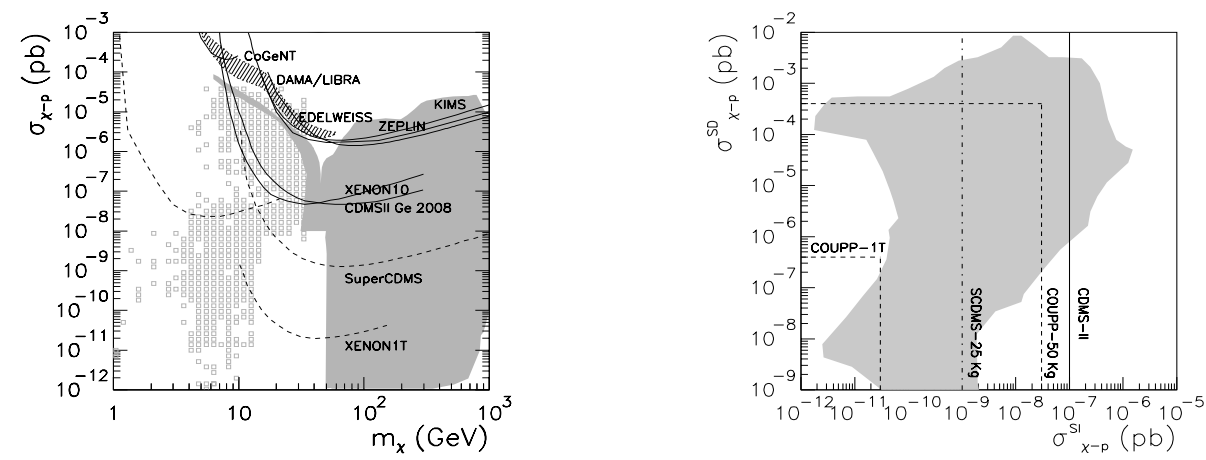

Fig. 1. Left) Theoretical predictions for the spin-independent contribution of the neutralinoproton scattering cross-section as a function of the neutralino mass. The gray area represents the results corresponding to a general scan in the MSSM, whereas the empty squares correspond to the predictions for light neutralinos in the NMSSM. ${ }^{5}$ Right) Spin-dependent versus spin-dependent contributions for a scan in general Supergravity. ${ }^{16}$

The detection prospects of the lightest neutralino in extended supersymmetric models is significantly different, due to the changes in the Higgs sector and the presence of new neutralino states. For example, this is the case of the Nextto-MSSM (NMSSM), in which the presence of very light Higgses (consistent with LEP constraints if they have a large singlet composition) can be used to obtain a sizable increase of the SI cross section. ${ }^{22,23,24}$ Moreover, in the NMSSM very light neutralinos (with masses below $10 \mathrm{GeV}$ ) are viable ${ }^{25}$ and can have very distinctive predictions for their direct detection, including, for example, smaller SI cross section than in the MSSM. ${ }^{5}$ The theoretical predictions for the SI cross section of neutralinos with $m_{\chi_{1}^{0}} \leq 30 \mathrm{GeV}$ are plotted in Fig. 1. Since the singlet component of the neutralino does not couple to the $Z$ boson or to squarks the theoretical predictions for the SD cross section remain the same as in the MSSM.

There is yet another viable supersymmetric WIMP candidate for dark matter, the lightest sneutrino. The left-handed sneutrino in the MSSM is excluded, since its sizable coupling to the $Z$ boson implies that they either annihilate too rapidly, or have large scattering cross sections already excluded by direct searches. ${ }^{26}$ Several models have been proposed to revive sneutrino DM by reducing its coupling with the Z-boson. This can be achieved by introducing a mixture of left- and right-handed sneutrinos, ${ }^{29,30,27,28}$ or by considering a purely right-handed sneutrino in models with an extended gauge sector ${ }^{31}$ or Higgs sector ${ }^{33,32}$ such as the NMSSM. ${ }^{34}$ In the first class of models, the elastic scattering of sneutrinos with quarks would take place through the $t$-exchange of $Z$ bosons, whereas in the second class it would mostly be due to the exchange of Higgs bosons. The resulting SI cross section in these cases can be within the reach of future detectors for a wide range of sneutrino masses. ${ }^{34}$ Being a scalar particle, the SD cross section vanishes for the sneutrino. 

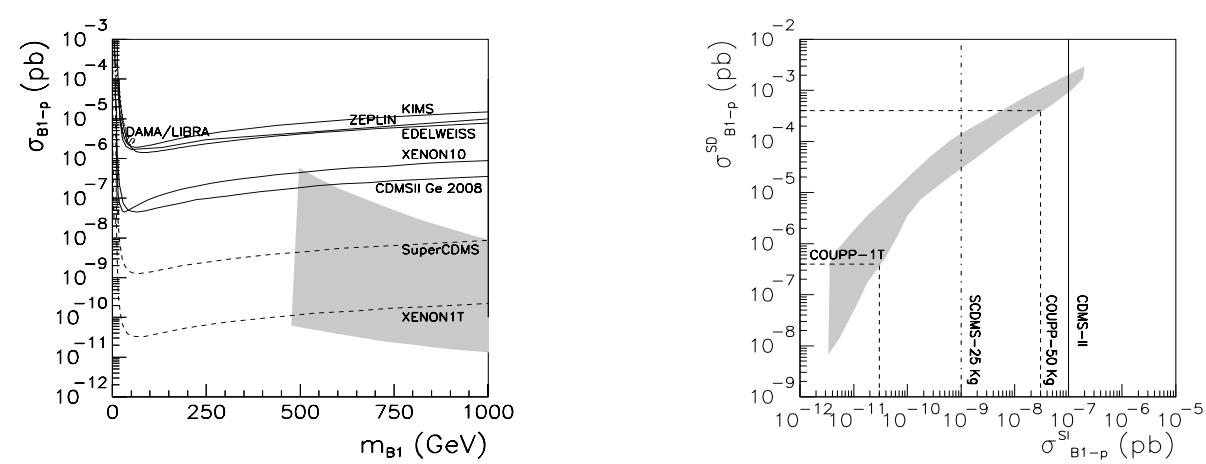

Fig. 2. Left) Theoretical predictions for the spin-independent contribution of the $B_{0}^{(1)}$ scattering cross-section as a function of its mass. Right) Spin-dependent versus spin-dependent contributions.

\subsection{Models with universal extra dimensions}

An interesting alternative arises in theories with Universal Extra Dimensions (UED), in which all fields are allowed to propagate in the bulk. ${ }^{35}$ Realistic models in which chiral fermions are introduced require a special compactification of the extra dimension, which implies a discrete symmetry, Kaluza-Klein parity. In this case, the Lightest Kaluza-Klein Particle (LKP) is stable and a therefore a potential DM candidate. The LKP is usually the first KK excitation of the hypercharge gauge boson, ${ }^{36,37} B_{0}^{(1)}$. In absence of spectral degeneracies the correct relic density can be obtained for masses in the $850-900 \mathrm{GeV}$ range. ${ }^{37}$ However, due to the quasidegenerate nature of the KK spectrum, this result is significantly modified due to coannihilations $^{38,39}$ with other KK-level modes.

The elastic scattering of $B_{0}^{(1)}$ with quarks takes place through the exchange of KK quarks along $t$ and $s$ channels, which contribute to both the SD and SI cross section, and a Higgs exchange along a $t$-channel which only gives a SI contribution. ${ }^{41,40}$ The theoretical predictions for the elastic scattering cross sections of $B_{0}^{(1)}$ are very dependent on the mass splitting between it and the KK quark, $\Delta_{q}$. In particular, both the SI and SD contributions increase when $\Delta_{q}$ becomes small, as a consequence of the enhancement of the contribution from KK quarks. This is particularly relevant since in UED the splittings between the masses are only induced by radiative corrections. ${ }^{36}$ The SI cross section can also be larger in the presence of light Higgses and for small LKP masses. However the Higgs mass is generally larger than in the MSSM and this contribution is suppressed.

The theoretical predictions for the SI cross section of $B_{0}^{(1)}$ can be as large as $\sigma^{S I} \approx 10^{-6} \mathrm{pb}$ for masses ranging from $500 \mathrm{GeV}$ to $1 \mathrm{TeV}$, when $\Delta_{q} \approx 0.01$, for which the correct relic density can be obtained. ${ }^{38,39}$ Under the same conditions, the predicted SD cross section are smaller and, for masses up to $1 \mathrm{TeV}$, ton-scale detectors would be required to detect them. These predictions are illustrated in Fig. 2, where the data has been taken from Refs. 42 and 16. 

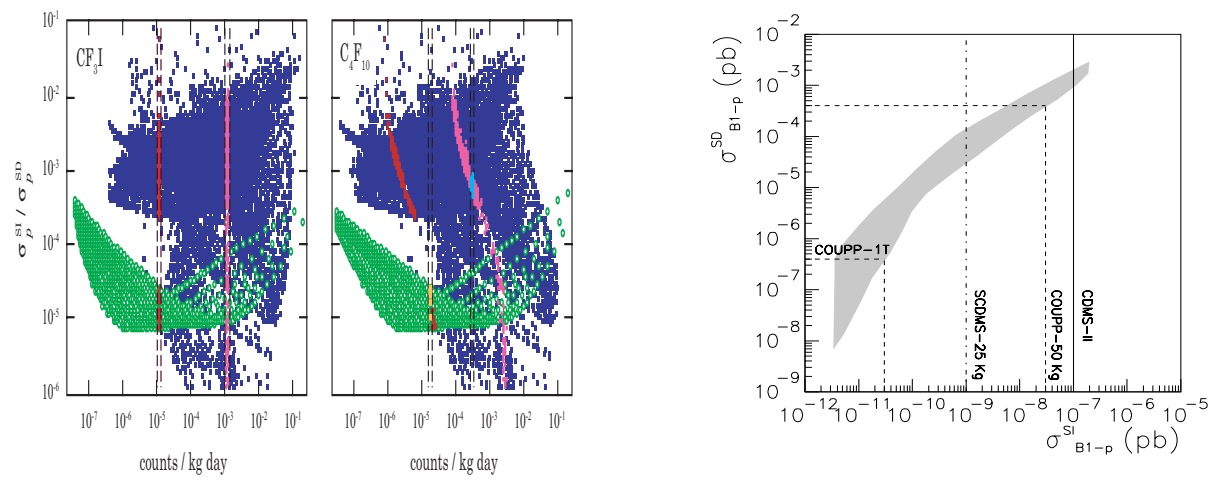

Fig. 3. (Color online) (Left) The detection with a given target such as $\mathrm{CF}_{3} \mathrm{I}$ can only loosely constrain models for neutralinos (blue) and LKP (green) in the $\sigma_{p}^{\mathrm{SI}} / \sigma_{p}^{\mathrm{SD}}$ versus count-rate plane. Red (magenta) dots show the points consistent with a measurement of $\sim 10^{-5}\left(10^{-3}\right) \mathrm{counts} / \mathrm{kg}$ day. Right) measurement of the event rate in a second target such as $\mathrm{C}_{4} \mathrm{~F}_{10}$ reduces the number of allowed models, potentially allowing discrimination between the neutralino and the LKP. ${ }^{16}$

\subsection{Little Higgs models}

Finally, another interesting possibility are Little Higgs models. These were proposed as an extension of the SM in which the stability of the mass of the Higgs boson is explained through a process of collective symmetry breaking at the TeV scale. These theories therefore imply the inclusion of new particles (gauge bosons, fermions and scalars) at that scale. These would considerably affect low-energy phenomenology through their contribution to low-energy observables. Indeed, in order to fulfil the constraints on precision electrweak fits a discrete symmetry has to be introduced (T-parity) which acts in a similar way to R-parity in Supersymmetric theories. It forbids dangerous diagrams and as a byproduct, renders the lightest T-odd particle (LTP) stable, thus making it another candidate for dark matter. ${ }^{43,44}$

The LTP is usually the partner of the hypercharge gauge boson $B_{H} \cdot{ }^{45,46,47} \mathrm{Its}$ scattering cross section with nuclei receives SI contributions via Higgs and heavy quark exchange, the latter being the only contribution to the SD part. ${ }^{48}$ Thus, the resulting expressions are very similar to the case of KK dark matter in UED. However, unlike the UED scenario, there is no reason for the heavy quarks to be degenerate in mass with the LTP. This, together with the smallness of the heavy quark Yukawa couplings implies that their scattering cross-sections are very suppressed. The SI cross section, being dominated by the Higgs exchange $t$-channel, increases slightly when the Higgs mass is small but is generally below $10^{-10} \mathrm{pb}$. The theoretical predictions for the SD cross section are also very small. In summary, the direct detection of the LTP is much more difficult than the SUSY and UED cases. 


\section{Discrimination of WIMP Candidates}

Current experiments are already probing the masses and cross-sections predicted for various WIMP candidates. Furthermore future experiments will be sensitive to a substantial fraction of the parameter space. If any of these experiments succeeds in detecting dark matter particles, the next objective will be to identify its particle nature. In this sense, the combination from the different techniques (direct, indirect and collider searches) could provide complementary information on the WIMP properties and would therefore be a crucial tool.

For example, the simultaneous measurement of both the SI and SD dark matter couplings, with experiments sensitive to both signals, can provide very valuable information. ${ }^{16}$ This is illustrated in Fig. 3, where the ratio of SI to SD cross section is plotted for the neutralino and LKP cases versus the event rate for two complementary targets of the COUPP detector. The measurement of an event rate in a single detector reduces the number of allowed models, but does not provide information on the nature of the dark matter detected. A subsequent detection using a second complementary target substantially reduces the allowed range of coupling parameters, and allows, in most cases, an effective discrimination between neutralino and LKP candidates. This analysis can be extended to other dark matter candidates. Also, in order to eliminate the large astrophysical and theoretical uncertainties which affect the dark matter rates, the ratio of WIMP-proton and WIMP-neutron amplitudes can be used ${ }^{49}$ and compared for different target materials.

Moreover, exploiting the dependence of the recoil energy spectrum with the WIMP mass, and combining the information from two experiments with different targets, the WIMP mass might be determined. ${ }^{50,51,52}$ Combining this information with hypothetical results from indirect searches, information on the spin of the dark matter particle can even be obtained. ${ }^{53}$ Needless to say, collider searches could provide further information on the WIMP properties, for example, allowing a more precise measurement of its mass which could then be used in combination with the results from direct and indirect detection. ${ }^{54}$

\section{Conclusions}

The impressive advances in dark matter detection will allow us to explore very efficiently the parameter spaces of many models proposing dark matter candidates. This is particularly interesting for the case of WIMPs (appearing in theories such as Supersymmetry, Universal Extra Dimensions models or Little Higgs Theories), since the combination of data from direct, indirect and collider searches could be used for a better determination of its properties and ultimately for its identification.

\section{Acknowledgments}

Work supported by the program "Juan de la Cierva" of the Spanish MEC, the MEC project FPA2006-01105, the EU network MRTN-CT-2006-035863, the ENTApP 
Network of the ILIAS project RII3-CT-2004-506222 and the project HEPHACOS P-ESP-00346 of the Comunidad de Madrid.

\section{References}

1. J. Dunkley et al., Astrophys. J. Suppl. 180, 306-329 (2009).

2. R. Bernabei et al., Eur. Phys. J. C56, 333-355 (2008).

3. Z. Ahmed et al., Phys. Rev. Lett. 102, 011301 (2009).

4. J. Angle et al., Phys. Rev. Lett. 100, 021303 (2008).

5. C. E. Aalseth et al., Phys. Rev. Lett. 101, 251301 (2008).

6. E. Behnke et al., Science 319, 933-936 (2008).

7. H. S. Lee et al., Phys. Rev. Lett. 99, 091301 (2007).

8. O. Adriani et al., arXiv:0810.4995.

9. S. Profumo, arXiv:0812.4457.

10. G. Jungman, M. Kamionkowski and K. Griest, Phys. Rept. 267, 195-373 (1996).

11. C. Muñoz, Int. J. Mod. Phys. A19, 3093-3170 (2004).

12. M. Drees and M. M. Nojiri, Phys. Rev. D47, 4226-4232 (1993).

13. J. R. Ellis, A. Ferstl and K. A. Olive, Phys. Lett. B481, 304-314 (2000).

14. G. B. Gelmini, P. Gondolo and E. Roulet, Nucl. Phys. B351, 623-644 (1991).

15. M. Srednicki and R. Watkins, Phys. Lett. B225, 140 (1989).

16. G. Bertone, D. G. Cerdeño, J. I. Collar and B. C. Odom, Phys. Rev. Lett. 99, 151301 (2007).

17. Y. G. Kim, T. Nihei, L. Roszkowski and R. Ruiz de Austri, JHEP 12, 034 (2002).

18. J. R. Ellis, K. A. Olive, Y. Santoso and V. C. Spanos, Phys. Rev. D71, 095007 (2005).

19. A. Bottino, N. Fornengo and S. Scopel, Phys. Rev. D67, 063519 (2003).

20. A. Bottino, F. Donato, N. Fornengo and S. Scopel, Phys. Rev. D69, 037302 (2004).

21. D. G. Cerdeño and C. Muñoz, JHEP 10, 015 (2004).

22. D. G. Cerdeño, C. Hugonie, D. E. Lopez-Fogliani, C. Muñoz and A. M. Teixeira, JHEP 12, 048 (2004).

23. D. G. Cerdeño, E. Gabrielli, D. E. Lopez-Fogliani, C. Muñoz and A. M. Teixeira, JCAP 0706, 008 (2007).

24. C. Hugonie, G. Belanger and A. Pukhov, JCAP 0711, 009 (2007).

25. J. F. Gunion, D. Hooper and B. McElrath, Phys. Rev. D73, 015011 (2006).

26. T. Falk, K. A. Olive and M. Srednicki, Phys. Lett. B339, 248-251 (1994).

27. C. Arina and N. Fornengo, JHEP 11, 029 (2007).

28. C. Arina, F. Bazzocchi, N. Fornengo, J. C. Romao and J. W. F. Valle, Phys. Rev. Lett. 101, 161802 (2008).

29. N. Arkani-Hamed, L. J. Hall, H. Murayama, D. Tucker-Smith and N. Weiner, Phys. Rev. D64, 115011 (2001).

30. D. Hooper, J. March-Russell and S. M. West, Phys. Lett. B605, 228-236 (2005).

31. H.-S. Lee, K. T. Matchev and S. Nasri, Phys. Rev. D76, 041302 (2007).

32. F. Deppisch and A. Pilaftsis, JHEP 10, 080 (2008).

33. B. Garbrecht, C. Pallis and A. Pilaftsis, JHEP 12, 038 (2006).

34. D. G. Cerdeño, C. Muñoz and O. Seto, Phys. Rev. D79, 023510 (2009).

35. T. Appelquist, H.-C. Cheng and B. A. Dobrescu, Phys. Rev. D64, 035002 (2001).

36. H.-C. Cheng, K. T. Matchev and M. Schmaltz, Phys. Rev. D66, 036005 (2002).

37. G. Servant and T. M. P. Tait, Nucl. Phys. B650, 391-419 (2003).

38. F. Burnell and G. D. Kribs, Phys. Rev. D73, 015001 (2006).

39. K. Kong and K. T. Matchev, JHEP 01, 038 (2006).

40. H.-C. Cheng, J. L. Feng and K. T. Matchev, Phys. Rev. Lett. 89, 211301 (2002). 
41. G. Servant and T. M. P. Tait, New J. Phys. 4, 99 (2002).

42. S. Arrenberg, L. Baudis, K. Kong, K. T. Matchev and J. Yoo, Phys. Rev. D78, 056002 (2008).

43. N. Arkani-Hamed, A. G. Cohen, E. Katz and A. E. Nelson, JHEP 07, 034 (2002).

44. J. Hubisz and P. Meade, Phys. Rev. D71, 035016 (2005).

45. H.-C. Cheng and I. Low, JHEP 09, 051 (2003).

46. H.-C. Cheng and I. Low, JHEP 08, 061 (2004).

47. I. Low, JHEP 10, 067 (2004).

48. A. Birkedal, A. Noble, M. Perelstein and A. Spray, Phys. Rev. D74, 035002 (2006).

49. G. Belanger, E. Nezri and A. Pukhov, Phys. Rev. D79, 015008 (2009).

50. A. M. Green, JCAP 0708, 022 (2007).

51. A. M. Green, JCAP 0807, 005 (2008).

52. M. Drees and C.-L. Shan, JCAP 0806, 012 (2008).

53. M. Beltran, D. Hooper, E. W. Kolb and Z. C. Krusberg, , arXiv:0808.3384.

54. N. Bernal, A. Goudelis, Y. Mambrini and C. Munoz, JCAP 0901, 046 (2009). 